\title{
Distinct local structure of superconducting $\mathrm{Ca}_{10} M_{4} \mathrm{Ass}_{8}\left(\mathrm{Fe}_{2} \mathrm{As}_{2}\right)_{5}(M=\mathrm{Pt}$, Ir $)$
}

\author{
E. Paris, ${ }^{1}$ T. Wakita, ${ }^{2}$ O. Proux,${ }^{3,4}$ T. Yokoya,${ }^{2}$ K. Kudo, ${ }^{2}$ D. Mitsuoka, ${ }^{2}$ T. Kimura, ${ }^{2}$ K. Fujimura, ${ }^{2}$ N. Nishimoto, ${ }^{2}$ S. Ioka, ${ }^{2}$ \\ M. Nohara, ${ }^{2}$ T. Mizokawa, ${ }^{5}$ and N. L. Saini ${ }^{1}$ \\ ${ }^{1}$ Dipartimento di Fisica, Universitá di Roma "La Sapienza" - Piazzale Aldo Moro 2, 00185 Roma, Italy \\ ${ }^{2}$ Research Institute for Interdisciplinary Science (RIIS), Okayama University, Okayama 700-8530, Japan \\ ${ }^{3}$ Observatoire des Sciences de l'Univers de Grenoble (OSUG), UMR CNRS 832, Université Grenoble-Alpes, 38041 Grenoble Cedex 9 , France \\ ${ }^{4}$ BM30B/CRG-FAME, ESRF, Polygone Scientifique Louis Néel, 71 avenue des Martyrs, 38000 Grenoble, France \\ ${ }^{5}$ Department of Applied Physics, Waseda University, Tokyo 169-8555, Japan \\ (Received 6 June 2017; revised manuscript received 15 November 2017; published 15 December 2017)
}

\begin{abstract}
We have studied the local structure of superconducting $\mathrm{Ca}_{10} \mathrm{Pt}_{4} \mathrm{As}_{8}\left(\mathrm{Fe}_{2} \mathrm{As}_{2}\right)_{5}$ ( $\mathrm{Pt10418)}$ and $\mathrm{Ca}_{10} \mathrm{Ir}_{4} \mathrm{As}_{8}\left(\mathrm{Fe}_{2} \mathrm{As}_{2}\right)_{5}$ (Ir10418) iron arsenides, showing different transition temperatures $\left(T_{c}=38\right.$ and $16 \mathrm{~K}$, respectively), by polarized Fe $K$-edge extended x-ray absorption fine-structure measurements. Despite the similar average crystal structures, the local structures of the $\mathrm{FeAs}_{4}$ tetrahedra in the two compounds are found to be very different. The $\mathrm{FeAs}_{4}$ in Pt10418 is close to a regular tetrahedron, while it deviates largely in Ir10418. The $\mathrm{Fe}-\mathrm{Fe}$ correlations in the two compounds are characterized by similar bond-length characteristics; however, the static disorder in Pt10418 is significantly lower than that in Ir10418. The results suggest that the optimized local structure and reduced disorder are the reasons for higher $T_{c}$ and well-defined electronic states in Pt10418 unlike Ir10418 showing the coexistence of glassy and normal electrons at the Fermi surface, and hence provide direct evidence of the local-structure-driven optimization of the electronic structure and superconductivity in iron arsenides.
\end{abstract}

DOI: 10.1103/PhysRevB.96.224507

\section{INTRODUCTION}

The discovery of superconductivity in layered iron arsenides [1] was followed by stimulating efforts to understand the mechanism of superconductivity in them and to explore new layered systems with desirable superconducting properties for their possible applications. Generally, these layered arsenides are characterized by tetrahedrally coordinated $\mathrm{FeAs}_{4}$ metallic layers intercalated by a nonmetallic spacer layer, different for different families of iron-based superconducting arsenides [2,3]. Among these, the skutterudite-type layered arsenides $\mathrm{Ca}_{10} M_{n} \mathrm{As}_{8}\left(\mathrm{Fe}_{2} \mathrm{As}_{2}\right)_{5}(M=\mathrm{Pt}$, Ir) are characterized by complex spacer layers [4-13] that would be conducting unlike the one in most of the iron-based superconductors. The $\mathrm{FeAs}_{4}$ active layers are stacked with $M_{n} \mathrm{As}_{8}$ layers, where $M=$ Pt or Ir and $n=3$ or 4 . Between the active and spacer layers, $\mathrm{Ca}$ atoms are intercalated in the structure. The conducting nature of the spacer layer in these systems is due to non-negligible contribution of $5 d$ electrons of $M(\mathrm{Pt}$ or Ir) at or near the Fermi level [11,12], responsible for the self-doping of carriers from the spacer layer through interlayer interaction [14].

Despite having a similar average structure, $\mathrm{Ca}_{10} \mathrm{Pt}_{4} \mathrm{As}_{8}\left(\mathrm{Fe}_{2} \mathrm{As}_{2}\right)_{5}$ (hereafter denoted as Pt10418) and $\mathrm{Ca}_{10} \mathrm{Ir}_{4} \mathrm{As}_{8}\left(\mathrm{Fe}_{2} \mathrm{As}_{2}\right)_{5}$ (Ir10418) with $n=4$ possess substantially different physical properties. For instance, Pt10418 shows a higher transition temperature $\left(T_{c} \sim 38 \mathrm{~K}\right)$ than $\operatorname{Ir} 10418\left(T_{c} \sim 16 \mathrm{~K}\right)$. In most of the iron arsenide superconductors, the magnitude of the superconducting transition temperature strongly depends on the spacer layer through the As height from the Fe-Fe layer (or As-Fe-As bond angle). This correlation seems unclear in the case of skutterudite-type structures in which the As height (or As-Fe-As bond angle) is argued to be insufficient to explain the difference in $T_{c}$ [4]. In addition, the normal-state electronic structure of the two systems, measured by angle-resolved photoemission spectroscopy (ARPES), is very different.
While the Pt10418 has [15] an electronic structure similar to the other iron-based superconductors, the Ir10418 shows [16] the coexistence of glassy electrons and normal electrons. It has been pointed out that under the tetragonal ligand field (for Pt or Ir in the spacer layer), the lower $e_{g}$ level is fully occupied in the $d^{8}$ configuration of $\mathrm{Pt}^{2+}$, while it is partially occupied in the $d^{7}$ configuration of $\operatorname{Ir}^{2+}$ [11]. As a result, $e_{g}$ orbitals of the Ir sites tend to have stronger hybridization with the apical As site that belongs to the active FeAs layer. Ir10418 has some Ir sites with pyramidal coordination affecting the As position in the FeAs layer and inducing a structural phase transition around $100 \mathrm{~K}$ [12]. Such a large difference in the electronic and structural properties of these arsenides indicates that apart from others, the short-range atomic correlations may have an important role in describing the physical properties of these materials.

Earlier, the local structure of Ir 10418 was studied [17] by a combined analysis of Fe $K$-edge and $\operatorname{Ir} L_{3}$-edge x-ray absorption spectroscopy to find some correlation with its peculiar electronic structure [16]. From the extended x-ray absorption fine-structure (EXAFS) study performed on polycrystalline samples of Ir10418, it was found that the Fe-As bonds are covalent as in the other iron-based superconductors [18], while the Fe-Fe structural networks are weak and contain excess local atomic disorder. It was concluded that the local disorder in the $\mathrm{Ir}_{4} \mathrm{As}_{8}$ layer could propagate into the active layer, making the Fe-Fe lattice relatively flexible. Here, we have studied the local structure of Pt10418 and Ir10418 comparatively to address the differing physical properties of these isostructural compounds. Since the Fermi surface of Fe-based materials is driven by $\mathrm{Fe} 3 d$ electrons, for a realistic comparison, we have used Fe $K$-edge EXAFS measured on single-crystal samples of the two systems. The experimental approach has been to use polarized EXAFS to study directional atomic displacements with enhanced $\mathrm{Fe}-\mathrm{Fe}$ signal in the polarization parallel to 
the $a b$ plane (the information on $\mathrm{Fe}-\mathrm{Fe}$ bond distance is not available in the $c$-axis polarized spectra and hence not measured). This experimental approach for the comparative study was also based on the fact that while Fe-As contribution in the unpolarized [17] and in-plane polarized EXAFS remains similar, the Fe-Fe contribution is amplified in the latter. Therefore, the chosen approach is a useful tool to quantify the differences in the $\mathrm{Fe}-\mathrm{As}$ and $\mathrm{Fe}-\mathrm{Fe}$ bond distances required to determine the As height (or As-Fe-As angle). We have found that the FeAs 4 network in Pt10418 is close to a regular tetrahedron, while the one in Ir10418 deviates largely from that. In addition, Fe-Fe correlations in the two compounds are characterized by a large static disorder in $\operatorname{Ir} 10418$ that is significantly lower in Pt10418. These results suggest that the reduced disorder and the optimized local structure of Pt10418 are the reasons for higher $T_{c}$ and well-defined electronic states.

\section{EXPERIMENTAL DETAILS}

X-ray absorption spectroscopy (XAS) measurements were performed at the beamline BM30B of the European Synchrotron Radiation Facility (ESRF) on single-crystal samples of $\mathrm{Ca}_{10} \mathrm{Pt}_{4} \mathrm{As}_{8}\left(\mathrm{Fe}_{2} \mathrm{As}_{2}\right)_{5}$ and $\mathrm{Ca}_{10} \mathrm{Ir}_{4} \mathrm{As}_{8}\left(\mathrm{Fe}_{2} \mathrm{As}_{2}\right)_{5}$. The details of the sample preparation and their characterization for the structural and transport properties are described elsewhere [11]. Single-crystal samples, with the crystallographic flat $a b$-plane surface, of the two systems were mounted in a continuous-flow He cryostat and temperature-dependent XAS measurements were carried out in fluorescence yield mode. For the measurements, normal incidence geometry (an angle $\sim 5^{\circ}$ off the sample normal) was used. In this geometry, the polarization of the incident beam is almost parallel to the $a b$ plane of the samples (i.e., $E \| a b$ ). The Fe $K$-edge absorption spectra were measured by detecting the Fe $K_{\alpha}$ fluorescence photons. The beam size (full width at half maximum) on the samples was $\sim 250 \mu \mathrm{m}(\mathrm{H}) \times 150 \mu \mathrm{m}(\mathrm{V})$. The obtained XAS spectra were corrected for the fluorescence self-absorption of the $K_{\alpha}$ photons using the FLUO algorithm embedded in ATHENA software [19] for the near-edge part. The fluorescence self-absorption corrected $\mathrm{x}$-ray absorption near-edge structure (XANES) spectra are compared in [20]. On the other hand, the algorithm developed by Tröger et al. was used to correct the EXAFS spectra [21].

\section{RESULTS AND DISCUSSION}

Figure 1 shows Fourier transforms (FTs) of the $k^{2}$-weighted polarized Fe $K$-edge EXAFS oscillations measured on Pt10418 and Ir10418 iron arsenides at a selected temperature of $60 \mathrm{~K}$. The EXAFS oscillations are shown as the inset. For a realistic comparison, the two samples were measured sequentially during the same experimental run and under the same experimental conditions. The EXAFS extraction was performed in the same way and the $k$ range used for both samples is the same ( 3 to $14 \AA^{-1}$ ). The FTs provide information on the atomic distribution around the Fe site. It should be mentioned that the position of the FT peaks does not appear at the actual bond distances since it should be corrected by the scattering phase shifts. Here, the FT peak structure appearing between 1.5-3.0 A contains two contributions: the one due to

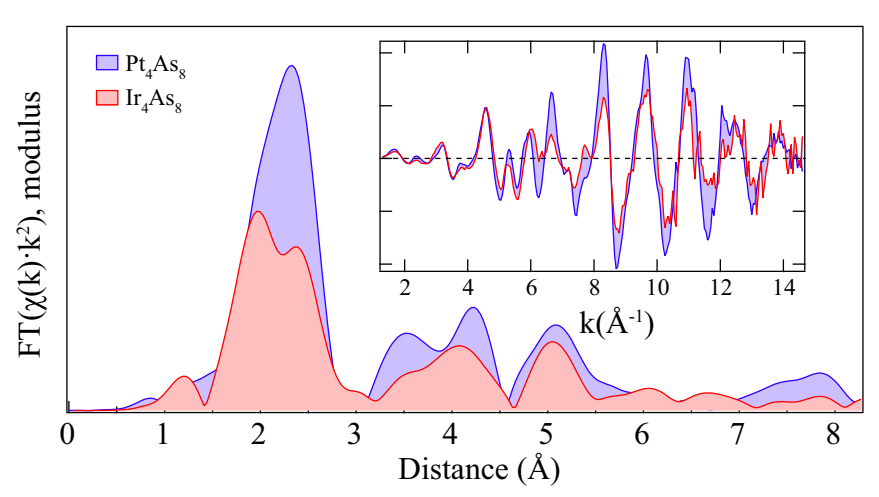

FIG. 1. Fourier-transformed magnitude of in-plane polarized Fe $K$-edge EXAFS oscillations extracted from the absorption spectra measured on Pt10418 $\left(\mathrm{Pt}_{4} \mathrm{As}_{8}\right)$ and $\operatorname{Ir} 10418\left(\mathrm{Ir}_{4} \mathrm{As}_{8}\right)$ at $T=60 \mathrm{~K}$ and not corrected by photoelectron phase shifts. The corresponding EXAFS oscillations are displayed as the inset.

Fe-As distances $(\sim 2.4 \AA)$ and the other due to Fe-Fe distances $(\sim 2.7 \AA)$ with respect to the polarization of the beam. The advantage of the in-plane polarization is evident from the FT of the EXAFS on Ir10418 showing a pronounced peak structure due to $\mathrm{Fe}-\mathrm{Fe}$ distance with respect to the one for unpolarized EXAFS on the powder sample of the same system [17]. Also, the differences in the local structure of the two systems are clear from both EXAFS oscillations and FTs, largely damped in Ir10418. Such a damping indicates that the local disorder in Ir10418 is much larger than that in Pt10418.

For a further characterization of the local disorder, we have performed the polarized Fe $K$-edge EXAFS measurements as a function of temperature that permit one to distinctly identify the static disorder from the thermal disorder. Figure 2 shows FTs of the EXAFS oscillations of Pt10418 at several temperatures. The EXAFS oscillations are displayed as the inset. The FTs of the temperature-dependent EXAFS on Ir10418 are shown in the Supplemental Material [20]. Apparently, the FT peak due to Fe-Fe distances in both systems decreases sharply with increasing temperature in comparison to the Fe-As peak, with the latter showing a smaller effect.

In order to extract the local structural parameters, the Fe $K$-edge EXAFS oscillations were modeled with two shells involving nearest-neighbor Fe-As distances $(\sim 2.4 \AA)$ and second-nearest-neighbor Fe-Fe distances $(\sim 2.7 \AA)$. The EXAFS modeling was carried out in the single scattering approximation using the standard EXAFS equation [22],

$$
\chi(k)=\sum_{i} \frac{N_{i} S_{0}^{2}}{k R_{i}^{2}} f_{i}\left(k, R_{i}\right) e^{-\frac{2 R_{i}}{\lambda}} e^{-2 k^{2} \sigma_{i}^{2}} \sin \left[2 k R_{i}+\delta_{i}(k)\right],
$$

where $N_{i}$ is the number of neighboring atoms at a distance $R_{i}, \delta_{i}$ is the phase shift, $f_{i}\left(k, R_{i}\right)$ is the backscattering amplitude, $\lambda$ is the photoelectron mean free path, and $\sigma_{i}^{2}$ represents the mean-square relative displacement (MSRD) parameter of the photoabsorber-backscatterer pairs. The $S_{0}^{2}$ is the EXAFS amplitude reduction factor due to many-body effects related to the losses occurring during the photoelectron propagation in the material (excitations as plasmons, electronhole pairs, etc.) and the intrinsic losses due to shake-up and 


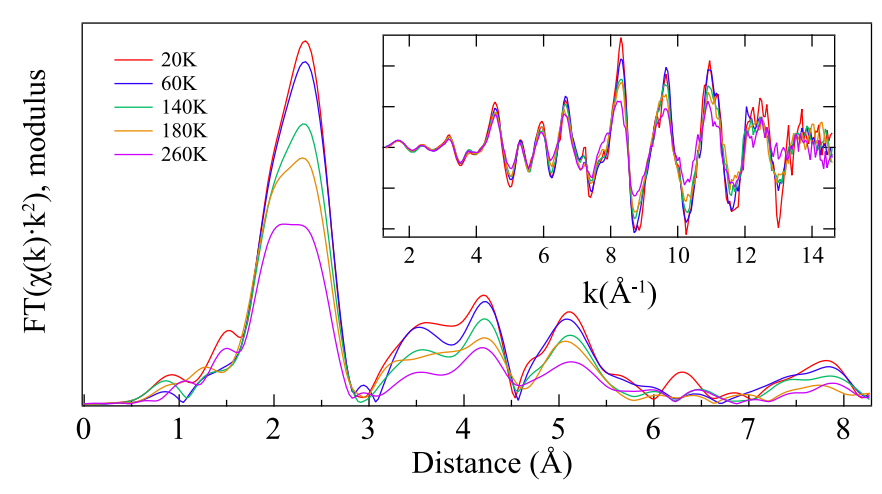

FIG. 2. Fourier transform magnitudes of the polarized Fe $K$-edge EXAFS on Pt10418 at several temperatures. The inset shows the corresponding EXAFS oscillations.

shake-off excitations created by the core hole in the absorption process. The effect of polarization was included for an effective number of neighbors to be $N_{i}=6 \cos ^{2}(\theta)$, where $\theta$ is the angle between the polarization vector and the direction of the distances from the photoabsorbing Fe (i.e., Fe-As and Fe-Fe distances). The ARTEMIS package [19] with the amplitude and phase factors calculated by the FEFF8 [23] was used for the model fits. In the starting model, the structural parameters deduced from x-ray diffraction measurements on Pt10418 and Ir10418 [11] were used. The effective number of near neighbors (respectively, 4 and 6 for Fe-As and $\mathrm{Fe}-\mathrm{Fe}$ distances) and the photoelectron energy zero $\left(E_{0}=0\right)$ were kept fixed, while the only parameters that were refined were the local bond distances and the associated $\sigma_{i}^{2}$. The $k$ range used for the EXAFS modeling was 3-14 $\AA^{-1}$, while the $R$ range was 1-3 $\AA$. Therefore, the number of independent data points, $2 \Delta k \Delta R / \pi$, was $\sim 14$ for the EXAFS model fits in which the fit parameters were four. The Fe $K$-edge EXAFS spectra along with the two-shell model fits are shown in Fig. 3.

The local bond distances in Pt10418, obtained by the EXAFS model fits, are shown in Fig. 4(a). The Fe-As bond $R_{F e-A s}$ is $2.419 \AA$ at room temperature and it shrinks down to $2.414 \AA$ at $20 \mathrm{~K}$. By linear fitting of $R_{F e-A s}(T)$ data, we obtain a rate of change $\partial R / \partial T \simeq 2.17 \times 10^{-5} \AA \mathrm{K}^{-1}$. The Fe-Fe distance is $\sim 2.758 \AA$ at room temperature with a small temperature dependence. While the Fe-As distances in the two compounds remain similar, the Fe-Fe local bond lengths in Ir10418 and Pt10418 are significantly different. In fact, the Fe-Fe bond distance in Ir 10418 is $R_{F e-F e} \sim 2.620 \AA$, differing by $\sim 0.14 \AA$ with respect to the one in Pt10418 and indicating the different local structure of the two compounds.

The anion height $\left(h_{p}\right)$ from the Fe-Fe layer and As-Fe-As bond angle $(\alpha)$ was estimated from the measured distances in the assumption of tetragonal symmetry using the following equations:

$$
\begin{aligned}
h_{p} & =\sqrt{R_{F e-A s}^{2}-\frac{1}{2} R_{F e-F e}^{2},} \\
\alpha & =\pi-2 \cos ^{-1}\left(\frac{R_{F e-F e}}{\sqrt{2} R_{F e-A s}}\right) .
\end{aligned}
$$

These parameters are shown in Figs. 4(c) and 4(b), respectively. Since the Fe-As bond distances are similar in Pt10418 and Ir10418 while the Fe-Fe distance differs substantially, the differences are to be reflected in both $h_{p}$ and $\alpha$. Indeed, the FeAs layer is found to be compressed in Pt10418 with respect to Ir10418. The value of $h_{p}$ in Pt10418 is found to be between 1.42 and $1.43 \AA$ in the whole temperature range, while it is $\sim 1.5 \AA$ in Ir10418. Accordingly, the As-Fe-As bond angle $\alpha$ is found to be $107-108^{\circ}$ in Pt10418 and $\sim 100-101^{\circ}$ in $\operatorname{Ir} 10418$. It is worth mentioning that an attempt was made to analyze the
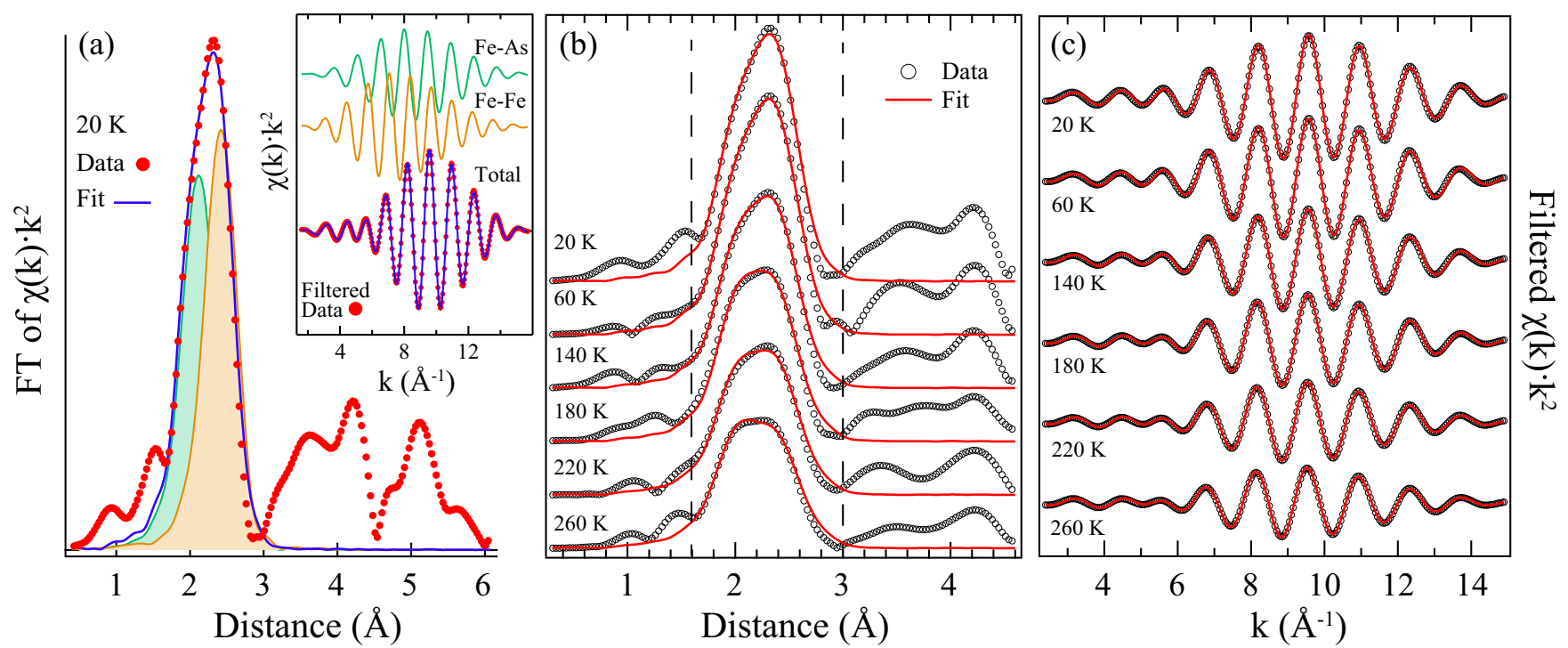

FIG. 3. (a) Fourier transform (FT) of the $\chi(k) k^{2}$ signal in $\mathrm{Ca}_{10} \mathrm{Pt}_{4} \mathrm{As}_{8}\left(\mathrm{Fe}_{2} \mathrm{As}_{2}\right)_{5}$ at the $\mathrm{Fe} K$ edge (red bubbles) compared with the best fit (blue line) for $T=20 \mathrm{~K}$. The real-space contributions of the Fe-As and Fe-Fe distances are shown as colored peaks. The inset shows the back Fourier transform of the spectra in the main panel taken between 1.5-3 $\AA$. The calculated $k$-space contributions of the Fe-As and Fe-Fe distances are shown with a vertical shift. (b) FTs of $\mathrm{Ca}_{10} \mathrm{Pt}_{4} \mathrm{As}_{8}\left(\mathrm{Fe}_{2} \mathrm{As}_{2}\right)_{5}$ (black bubbles) as a function of temperature are shown with the model fits (red solid lines). (c) Filtered $\chi(k) k^{2}$ [range of the back Fourier transform is indicated by vertical dashed lines in (b)] signals (data points) with the corresponding model fits (line) are shown. 

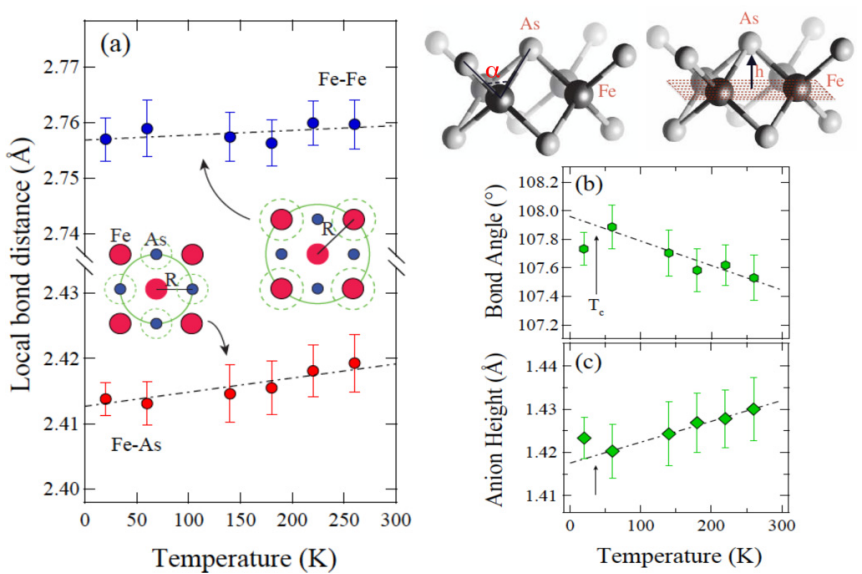

FIG. 4. (a) Temperature dependence of the Fe-As and Fe-Fe distances (pictorially represented) obtained from two-shell model fits of the Fe $K$-edge EXAFS for Pt10418. (b),(c) The $T$ dependence of As-Fe-As bond angle $(\alpha)$ and pnictogen height from the Fe-Fe layer $\left(h_{p}\right) . \alpha$ and $h_{p}$ have been computed considering a perfect tetragonal coordination (see text). The errors bars are estimated by analysis of different scans and considering the correlation between different fit parameters. A picture of the local structure is also shown, defining the two parameters (As-Fe-As angle $\alpha$ and the anion height).

data with reduced effective numbers for As to compensate for the effect of polarization, i.e., assuming the angle to be $108^{\circ}$ for Pt10418 (As coordination 3.9 instead of 4.0) and $101^{\circ}$ for Ir10418 (As coordination 3.6 instead of 4.0); however, within the experimental uncertainties, the resulting $\sigma_{i}^{2}$ for Fe-As and $\mathrm{Fe}-\mathrm{Fe}$ were found to be the same. These results on Ir10418 are partially consistent with the previous x-ray diffraction study by Katayama et al. on the same samples [12]. In the X-ray diffraction study, $h_{p}$ is $\sim 1.4 \AA$ when the Ir site just below the As site has square plane coordination. On the other hand, $h_{p}$ is $\sim 1.5 \AA$ if the Ir site has pyramidal coordination.

On the basis of average structure measurements, Ni et al. [4] have pointed out that Fe-based superconductors with skutterudite spacer layers do not follow the empirical relation of $h_{p}$ [24] and $\alpha$ [25] with $T_{c}$. Unlike those claims, we have found that the empirical rule of $h_{p}\left(T_{c}\right), \alpha\left(T_{c}\right)$ is highly robust and is valid also for these families of materials. Indeed, the difference of $h_{p}\left(\Delta h_{p} \sim 0.1 \AA\right)$ and $\alpha\left(\Delta \alpha \sim 7^{\circ}\right)$ between the two systems can easily explain the difference of $T_{c}$ in Pt10418 and Ir10418 ( $T_{c} \sim 38$ and $16 \mathrm{~K}$, respectively).

Figure 5(a) shows the temperature dependence of the $\sigma^{2}$, i.e., the mean-square relative displacements of the Fe-As and Fe-Fe distances for Pt10418. The temperature dependence of $\sigma^{2}(\mathrm{~T})$ can be described by the Einstein model $[23,26]$,

$$
\sigma^{2}(T)=\frac{\hbar^{2}}{2 k_{B} \mu \Theta_{E}} \operatorname{coth}\left(\frac{\Theta_{E}}{2 T}\right)+\sigma_{0}^{2},
$$

where $k_{B}$ is the Boltzmann constant, $\mu$ is the reduced mass of the considered absorber-backscatterer pair, and $\sigma_{0}^{2}$ is a offset related to the overall configurational disorder. Here, the $\sigma^{2}(\mathrm{~T})$ for the two bond lengths is well described by the Einstein model down to the lowest temperature measured. In the Einstein model, the atomic pair of atoms is treated as harmonic oscillators with corresponding Einstein temperatures
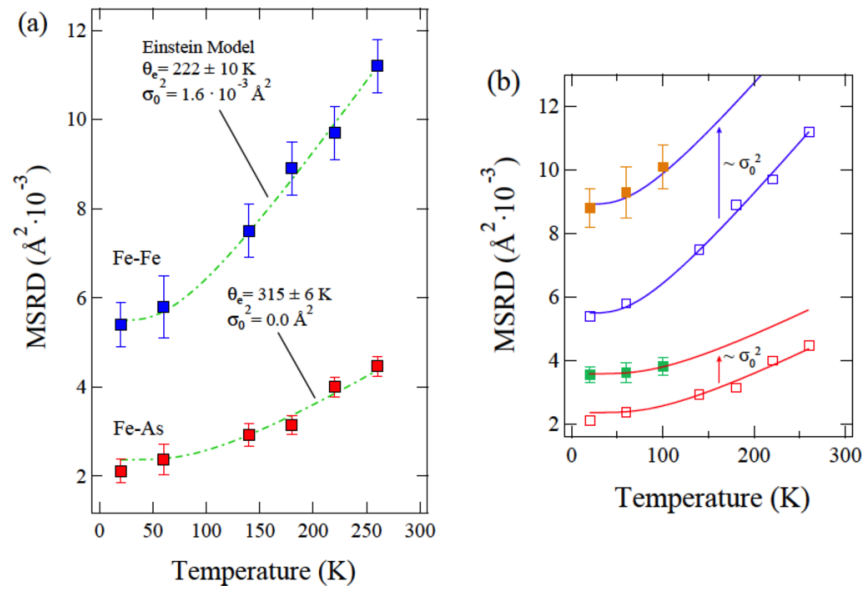

FIG. 5. (a) Temperature dependence of the mean-square relative displacement $\sigma^{2}$ for the Fe-As and Fe-Fe distances in Pt10418, obtained from the polarized Fe $K$-edge EXAFS analysis. The dashdotted line is the best-fit result of the Einstein model (see text). (b) Comparison between the $\sigma^{2}$ of Pt10418 [open symbols; same data as (a)] and Ir10418 (full symbols), measured in the low- $T$ region. The $\sigma^{2}$ obtained for Ir 10418 are compatible with the Einstein model obtained for Pt10418, however with a vertical shift that mimics a higher value of $\sigma_{0}^{2}$ in the former.

$\left(\Theta_{E}=\omega_{E} / k_{B}\right.$, with $\omega_{E}$ being Einstein frequency and $k_{B}$ the Boltzmann constant) that depend on the bonding. The $\Theta_{E}$, characterizing bond strength, increases with bond stiffness. The bond-stiffness parameter $\left(\kappa=\mu \omega_{E}^{2}, \mu\right.$ is reduced mass of the atomic pairs) is a measure of the effective bond-stretching force constant that depends on the extent of the thermal displacements of the pair of atoms, as well as on their correlation. In other words, the bond strength can be conceived as the restoring force for displacement of the equilibrium interatomic distance. From the temperature-dependent EXAFS, we have determined $\Theta_{E}$ using the model fits shown in Fig. 5(a). The Einstein temperatures for Pt10418 are found to be $\Theta_{E}=318 \pm 16 \mathrm{~K}$ and $\Theta_{E}=224 \pm 10 \mathrm{~K}$, respectively, for the Fe-As and Fe-Fe distances. The corresponding bond stiffness $\left(\kappa=\mu \omega^{2}\right)$ parameters are $\kappa_{F e A s}=5.7 \pm 0.6 \mathrm{eV} \AA^{-2}$ and $\kappa_{F e F e}=2.5 \pm 0.2 \mathrm{eV} \AA^{-2}$, respectively.

The Einstein temperatures of the two bonds for Pt10418 are found to be similar to those for Ir10418, with the latter being consistent with an earlier study on a polycrystalline sample [17]. This can be seen in Fig. 5(b) comparing the temperature dependence of $\sigma^{2}(\mathrm{~T})$ for the two systems determined from the EXAFS spectra obtained in similar conditions and analyzed in a similar way. From the comparison between the two, it is clear that the Ir10418 data is well described by the similar Einstein temperature but with different $\sigma_{0}^{2}$ representing the static configurational disorder. The Fe-As pair for the two compounds shows the same $\Theta_{E}$ within the experimental uncertainties, similar to the typical values found for iron pnictides. On the other hand, although the $\Theta_{E}$ for the $\mathrm{Fe}-\mathrm{Fe}$ bond is the same for Ir10418 and Pt10418, it shows considerable difference with respect to other compounds of the iron-based family as $\mathrm{LaFeAsO}_{1-x} \mathrm{~F}_{x}$ [27-29]. Similar Einstein temperatures for Ir 10418 and Pt10418 indicate similar 
TABLE I. Comparison of the local-structure parameters of $\mathrm{Ca}_{10} M_{4} \mathrm{As}_{8}\left(\mathrm{Fe}_{2} \mathrm{As}_{2}\right)_{5}(M=\mathrm{Pt}$,Ir).

\begin{tabular}{lccr}
\hline \hline & $h_{p}($ As height $)(\AA)$ & $\alpha($ As-Fe-As angle $)$ & $\sigma_{0}^{2}\left(\AA^{2}\right)(\mathrm{Fe}-\mathrm{Fe})$ \\
\hline $\mathrm{Ca}_{10} \mathrm{Pt}_{4} \mathrm{As}_{8}\left(\mathrm{Fe}_{2} \mathrm{As}_{2}\right)_{5}$ & $\sim 1.43$ & $\sim 108^{\circ}$ & $1.6 \times 10^{-3}$ \\
$\mathrm{Ca}_{10} \mathrm{Ir}_{4} \mathrm{As}_{8}\left(\mathrm{Fe}_{2} \mathrm{As}_{2}\right)_{5}$ & $\sim 1.53$ & $\sim 101^{\circ}$ & $5.1 \times 10^{-3}$ \\
\hline \hline
\end{tabular}

local bond dynamics, however with very different $\sigma_{0}^{2}$. The parameters of the local structure for the two compounds are also summarized in Table I. It is worth mentioning that anisotropic distribution of As may affect the $\sigma_{0}^{2}$ of the Fe-As distance; however, this should not have any substantial impact on the present findings revealing an overall increase in the configurational disorder in $\operatorname{Ir} 10418$ given by higher $\sigma_{0}^{2}$ values.

\section{CONCLUSIONS}

In summary, we have carried out a comparative study of the local structure of the $\mathrm{Ca}_{10} M_{4} \mathrm{As}_{8}\left(\mathrm{Fe}_{2} \mathrm{As}_{2}\right)_{5}$ system, with $M=\mathrm{Ir}$ and $\mathrm{Pt}$. The results provide key information on the local distortions and disorder that are different in the two systems. On the basis of these results, it is plausible to conclude that local disorder in the Fe-Fe layer, driven by the spacer layer, is responsible for the glassy nature of electrons in the $M=\mathrm{Ir}$ system [16], unlike normal electrons in the $M=\operatorname{Pt}$ system [15]. This difference in local disorder, combined with the large local distortion of the $\mathrm{FeAs}_{4}$ tetrahedron in Ir10418, has direct implication in the different $T_{c}$ observed in the two systems.

\section{ACKNOWLEDGMENTS}

We thank the ESRF staff for the assistance during the measurements. A part of this work was performed at the Advanced Science Research Center, Okayama University. This work was partly supported by Grants-in-Aid for Scientific Research (Grants No. $15 \mathrm{H} 05886$ and No. 16K05451), the Program for Promoting the Enhancement of Research Universities from MEXT, and the Program for Advancing Strategic International Networks to Accelerate the Circulation of Talented Researchers from JSPS. The work is a part of the executive protocol of the general agreement for cooperation between the Sapienza University of Rome and Okayama University. The work at the Sapienza University is partially supported by PRIN2012 (Grant No. 2012X3YFZ2).
[1] Y. Kamihara, T. Watanabe, M. Hirano, and H. Hosono, J. Am. Chem. Soc. 130, 3296 (2008).

[2] H. Hosono and K. Kuroki, Physica C 514, 399 (2015).

[3] D. C. Johnston, Adv. Phys. 59, 803 (2010).

[4] N. Ni, J. M. Allred, B. C. Chan, and R. J. Cava, Proc. Natl. Acad. Sci. USA 108, E1019 (2011).

[5] S. Kakiya, K. Kudo, Y. Nishikubo, K. Oku, E. Nishibori, H. Sawa, T. Yamamoto, T. Nozaka, and M. Nohara, J. Phys. Soc. Jpn. 80, 093704 (2011).

[6] M. Nohara, S. Kakiya, K. Kudo, Y. Oshiro, S. Araki, T. C. Kobayashi, K. Oku, E. Nishibori, and H. Sawa, Solid State Commun. 152, 635 (2012).

[7] T. Stürzer, G. Derondeau, and D. Johrendt, Phys. Rev. B 86, 060516(R) (2012).

[8] Z. J. Xiang, X. G. Luo, J. J. Ying, X. F. Wang, Y. J. Yan, A. F. Wang, P. Cheng, G. J. Ye, and X. H. Chen, Phys. Rev. B 85, 224527 (2012).

[9] M. Neupane, C. Liu, S. Y. Xu, Y. J. Wang, N. Ni, J. M. Allred, L. A. Wray, N. Alidoust, H. Lin, R. S. Markiewicz, A. Bansil, R. J. Cava, and M. Z. Hasan, Phys. Rev. B 85, 094510 (2012).

[10] X. P. Shen, S. D. Chen, Q. Q. Ge, Z. R. Ye, F. Chen, H. C. Xu, S. Y. Tan, X. H. Niu, Q. Fan, B. P. Xie, and D. L. Feng, Phys. Rev. B 88, 115124 (2013).

[11] K. Kudo, D. Mitsuoka, M. Takasuga, Y. Sugiyama, K. Sugawara, N. Katayama, H. Sawa, H. S. Kubo, K. Takamori, M. Ichioka, T. Fujii, T. Mizokawa, and M. Nohara, Sci. Rep. 3, 3101 (2013).

[12] N. Katayama, K. Sugawara, Y. Sugiyama, T. Higuchi, K. Kudo, D. Mitsuoka, T. Mizokawa, M. Nohara, and H. Sawa, J. Phys. Soc. Jpn. 83, 113707 (2014).
[13] S. Kitagawa, S. Araki, T. C. Kobayashi, H. Ishii, K. Fujimura, D. Mitsuoka, K. Kudo, and M. Nohara, Phys. Rev. B 90, 224513 (2014).

[14] T. Berlijn, Phys. Rev. B 89, 104511 (2014).

[15] M. Sunagawa, R. Yoshida, T. Ishiga, K. Tsubota, T. Jabuchi, J. Sonoyama, S. Kakiya, D. Mitsuoka, K. Kudo, M. Nohara, K. Ono, H. Kumigashira, T. Oguchi, T. Wakita, Y. Muraoka, and T. Yokoya, J. Phys. Soc. Jpn. 84, 055001 (2015).

[16] K. Sawada, D. Ootsuki, K. Kudo, D. Mitsuoka, M. Nohara, T. Noda, K. Horiba, M. Kobayashi, K. Ono, H. Kumigashira, N. L. Saini, and T. Mizokawa, Phys. Rev. B 89, 220508(R) (2014).

[17] E. Paris, B. Joseph, A. Iadecola, C. Marini, K. Kudo, D. Mitsuoka, M. Nohara, T. Mizokawa, and N. L. Saini, Phys. Rev. B 90, 094508 (2014).

[18] A. Iadecola, S. Agrestini, M. Filippi, L. Simonelli, M. Fratini, B. Joseph, D. Mahajan, and N. L. Saini, Europhys. Lett. 87, 26005 (2009).

[19] B. Ravel and M. Newville, J. Synch. Rad. 12, 537 (2005).

[20] See Supplemental Material at http://link.aps.org/supplemental/ 10.1103/PhysRevB.96.224507 doi for information including $\mathrm{Fe}$ $K$-edge polarized EXAFS and Fourier transforms of Ir10418 at different temperatures. Fe $K$-edge x-ray absorption near-edge structure (XANES) spectra of the two materials are also shown, compared at $60 \mathrm{~K}$.

[21] L. Tröger, D. Arvanitis, K. Baberschke, H. Michaelis, U. Grimm, and E. Zschech, Phys. Rev. B 46, 3283 (1992).

[22] X-ray Absorption: Principles, Applications, Techniques of EXAFS, SEXAFS, XANES, edited by R. Prins and D. C. Koningsberger (Wiley, New York, 1988).

[23] See, e.g., a review by J. J. Rehr and R. C. Albers, Rev. Mod. Phys. 72621 (2000). 
[24] Y. Mizuguchi, Y. Hara, K. Deguchi, S. Tsuda, T. Yamaguchi, K. Takeda, H. Kotegawa, H. Tou, and Y. Takano, Supercond. Sci. Technol. 23, 054013 (2010).

[25] C. H. Lee, K. Kihou, A. Iyo, H. Kito, P. M. Shirage, and H. Eisaki, Solid State Commun. 152, 644 (2012).

[26] E. Sevillano, H. Meuth, and J. J. Rehr, Phys. Rev. B 20, 4908 (1979).
[27] C. J. Zhang, H. Oyanagi, Z. H. Sun, Y. Kamihara, and H. Hosono, Phys. Rev. B 81, 094516 (2010).

[28] B. Joseph, A. Iadecola, L. Malavasi, and N. L. Saini, J. Phys. Condens. Matter 23, 265701 (2011).

[29] B. Joseph, A. Iadecola, L. Simonelli, L. Maugeri, A. Martinelli, A. Palenzona, M. Putti, and N. L. Saini, Supercond. Sci. Technol. 26, 65005 (2013). 